ARTICLE

https://doi.org/10.1038/s41467-019-13503-9

\title{
Vibronic coherence evolution in multidimensional ultrafast photochemical processes
}

\author{
James D. Gaynor (D) ${ }^{1}$, Jason Sandwisch (D) ${ }^{1} \&$ Munira Khalil (iD ${ }^{1 \star}$
}

The complex choreography of electronic, vibrational, and vibronic couplings used by photoexcited molecules to transfer energy efficiently is remarkable, but an unambiguous description of the temporally evolving vibronic states governing these processes has proven experimentally elusive. We use multidimensional electronic-vibrational spectroscopy to identify specific time-dependent excited state vibronic couplings involving multiple electronic states, high-frequency vibrations, and low-frequency vibrations which participate in ultrafast intersystem crossing and subsequent relaxation of a photoexcited transition metal complex. We discover an excited state vibronic mechanism driving long-lived charge separation consisting of an initial electronically-localized vibrational wavepacket which triggers delocalization onto two charge transfer states after propagating for $\sim 600$ femtoseconds. Electronic delocalization consequently occurs through nonadiabatic internal conversion driven by a $50 \mathrm{~cm}^{-1}$ coupling resulting in vibronic coherence transfer lasting for $\sim 1$ picosecond. This study showcases the power of multidimensional electronic-vibrational spectroscopy to elucidate complex, non-equilibrium energy and charge transfer mechanisms involving multiple molecular coordinates.

\footnotetext{
${ }^{1}$ Department of Chemistry, University of Washington, Box 351700, Seattle, WA 98195, USA. *email: mkhalil@uw.edu
} 
P hotoexcited charge and energy transfer are essential steps in many natural and artificial light-harvesting processes ${ }^{1-4}$, which often rely on transition metal complexes with characteristically dense manifolds of charge transfer excited states $^{5-11}$. The orchestration of electronic, vibrational, and electronic-vibrational (vibronic) couplings that drive charge and energy transfer in light-harvesting complexes involve structural rearrangement, spin-vibronic interactions, and a dynamic solvent environment making these systems fundamentally interesting and challenging to understand. A wealth of experimental and computational studies on Ru-centered complexes have measured their photoexcited dynamics following singlet metalto-ligand charge transfer (MLCT) excitation, such as ultrafast intersystem crossing (ISC). These past studies have established that the excited triplet MLCT states can provide sufficiently longlived charge separation to yield a useful chemical potential for energy harvesting applications ${ }^{12-18}$. Yet, an essential open question remains: what intramolecular coordinates define the trajectory of a photoexcited complex's evolution into these desirable long-lived charge separated states?

Addressing this requires a continually advancing spectroscopic toolbox to move beyond measuring kinetics of photoexcited states toward building detailed molecular-level descriptions of important charge and energy transfer mechanisms utilized by transition metal complexes ${ }^{19-23}$. Exciting progress in experimental techniques is making investigations related to the following questions tractable: how do specific molecular coordinates control photoexcited energy transfer dynamics? What role do vibronic couplings and coherences play during photoexcited energy transfer and relaxation? Third-order nonlinear Fourier transform (FT) techniques such as two-dimensional (2D) electronic (ES) and 2D infrared (IR) spectroscopy have greatly advanced our understanding of coherent molecular phenomena in solution ${ }^{24-33}$. While vibronic information may be measured indirectly by $2 \mathrm{D}$ IR and ES spectroscopies, the recently developed 2D electronic-vibrational (2D EV) and 2D vibrational-electronic experiments directly access vibronic information through resonant interactions with electronic and IR-active vibrational transitions ${ }^{34-37}$.

In this article, we use multidimensional (2D and $3 \mathrm{D}) \mathrm{EV}$ spectroscopy to directly follow vibronic coherence evolution in a photoexcited $\mathrm{Ru}$-centered complex: the solar cell dye molecule, $\left[\mathrm{Ru}-(\mathrm{dcbpy})_{2}(\mathrm{NCS})_{2}\right]$ (dcbpy $=4,4^{\prime}$-dicarboxy-2,2' -bipyridine), commonly known as $\mathrm{N} 3$. We demonstrate $3 \mathrm{D} \mathrm{EV}$ spectroscopy (Fig. 1d), where vibronic couplings between three disparate molecular coordinates are resolved: electronic excitations $\left(\omega_{1}: 24,100-25,500 \mathrm{~cm}^{-1}\right)$, low-frequency vibrations $\left(0<\omega_{2}<0-833 \mathrm{~cm}^{-1}\right)$, and high-frequency vibrations $\left(\omega_{3}\right.$ : $\left.1250-1500 \mathrm{~cm}^{-1}\right)$. A real-time microscopic view of photochemical reactivity may be obtained by following the temporal evolution of the vibronic states measured in the $3 \mathrm{D} \mathrm{EV} \mathrm{spec-}$ trum. As depicted in Fig. 1d, vibrational coherences (red/yellow spheres, $\mathrm{L}_{1} / \mathrm{L}_{1}{ }^{\prime}$ ) can be directly identified by an electronically localized $3 \mathrm{D}$ EV feature, and its time-dependent behavior $\left(\mathrm{L}_{1}\right.$ (red) $\rightarrow \mathrm{L}_{1}{ }^{\prime}$ (yellow)) characterized through its $\omega_{2}$-dependent spectral phase. Population and coherence transfer (blue and purple spheres, $\mathrm{L}_{2}$ ) between vibronic states can follow internal conversion (IC) from the perspective of specific vibrational coordinates that may be directly involved. Vibronic coherences can also be identified through out-of-phase oscillatory dynamics between different $3 \mathrm{D} \mathrm{EV}$ features. In this way, the 3D FT experiment can unveil additional dark coordinates actively coupling the two motions measured in the $2 \mathrm{D}$ experiment, as well as coherences between molecular states facilitating energy transfer. While 3D FT studies have yielded exciting insight related to vibrations driving singlet fission ${ }^{38}$, quantum coherence in photosynthesis ${ }^{39}$, and tracking excited-state a

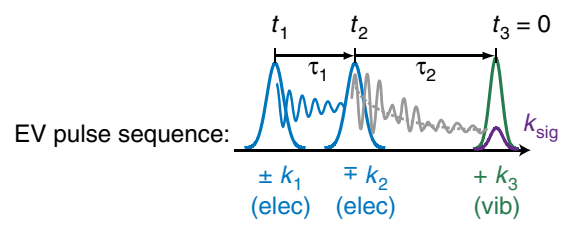

b
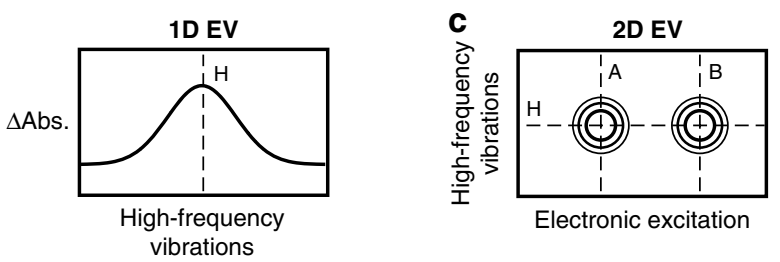

d

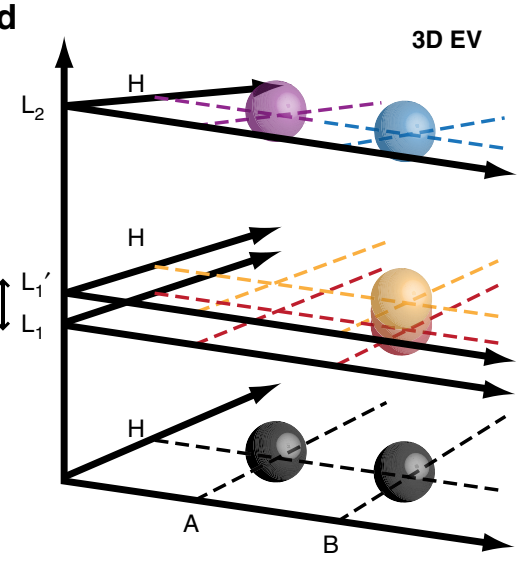

$A, B=$ electronic

$\mathrm{H}=$ high-freq. vibrations

$\mathrm{L}=$ low-freq. vibrations

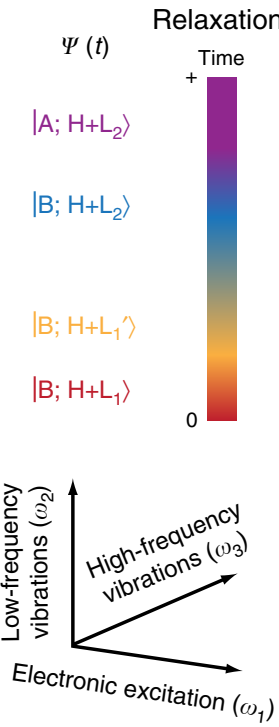

Fig. 1 Multidimensional Electronic-Vibrational (EV) Spectroscopy. In each technique, the pulse sequence (a) induces an electronic excitation (pumps: $\mathbf{k}_{1}, \mathbf{k}_{2}$ ) and measures a high-frequency vibrational response (probe: $\mathbf{k}_{3}$ ) in a sample. Consider a system where one high-frequency vibration $(H)$ vibronically couples to two electronic excited states $(A, B)$ and to two lowfrequency vibrations $\left(L_{1}, L_{2}\right)$ in $A$ and/or $B$. $b$ The pump-probe experiment (1D EV) measures the $\mathrm{H}$ vibrational spectrum $\left(\omega_{3}\right)$ and kinetics during its relaxation times $\left(\tau_{2}\right)$. At a given $\tau_{2}$ delay, $(\mathbf{c})$ the $2 \mathrm{D} \mathrm{EV}$ experiment resolves the electronic excitation spectrum $\left(\omega_{1}\right)$ for $\mathrm{H}$, defining the excited vibronic eigenstates $(\psi(t))$ by the couplings between $H$, the ground electronic state and either A or B. Collecting 2D EV spectra over a $\tau_{2}$ range measures population relaxation and coherent superpositions of eigenstates. The latter manifest as peak amplitude modulations; a FT over $\tau_{2}$ yields a (d) 3D EV spectrum where coherences (rainbow-colored spheres) separate in $\omega_{2}$ from population decays (black spheres) according to their beat frequency. The $\omega_{2}$ frequencies can indicate low-frequency vibrations $\left(L_{1}, L_{2}\right)$ coupled with vibronic eigenstates. Time-dependent 3D EV features, such as frequency shifts of low-frequency modes as generally indicated by $L_{1}$ moving to $L_{1}{ }^{\prime}$ in the $\omega_{2}$ frequency space, report on the temporal evolution and nonequilibrium relaxation of the vibronic eigenstates during photoexcited processes (depicted by the sphere coloration gradient).

photoisomerization ${ }^{40}$, no reported study to date has fully exploited the potential of 3D FT spectroscopy to most completely describe molecular eigenstates in terms of three energetically disparate molecular coordinates. Here, the complete capability of multidimensional EV spectroscopy is demonstrated by directly following vibronic coherence evolution in the photoexcited aqueous $\mathrm{N}^{4-}$ molecule (fully deprotonated N3) while it undergoes ultrafast ISC and nonadiabatic IC. An 
in-depth characterization of an excited-state vibrational wave packet is reported here in photoexcited $\mathrm{N}^{4-}$ as it propagates during the initial 600 femtoseconds (fs), answering an outstanding question about the wave packet's existence within the $\mathrm{N} 3$ triplet manifold ${ }^{12}$. Consequent energy transfer from the initial vibrational wave packet to a second coherence is observed, which propagates further for $\sim 1$ picosecond (ps). Interestingly, the electronic character of the evolving secondary coherence oscillates between two different MLCT states with $340 \pm 40 \mathrm{fs}$ periodicity. Our measurement identifies this as a vibronic coherence promoting nonadiabatic IC. We simulate the experimental result and extract the $50 \mathrm{~cm}^{-1}$ nonadiabatic coupling driving IC during the excited-state triplet relaxation. This study provides an unambiguous view of a photoexcited vibronic mechanism driving long-lived charge separation in a solar cell dye.

\section{Results}

The MLCT states and the high-frequency vibrational reporter. The aqueous $\mathrm{N}^{4-}$ has two ${ }^{1}$ MLCT electronic absorption bands (Supplementary Fig. 1) centered at $20,000 \mathrm{~cm}^{-1}(500 \mathrm{~nm})$ and $26,880 \mathrm{~cm}^{-1}(372 \mathrm{~nm})$. The ${ }^{1}$ MLCT absorption consisting of a dense manifold of MLCT excited states results in electron density with mixed Ru-(NCS $)_{2}$ character shifting to a dcbpy $\pi^{*}$ orbital ${ }^{8,41}$. The ${ }^{3}$ MLCT excited-state manifold is also dense, which facilitates ultrafast ISC on a timescale comparable with one or two vibrational periods of the ligand high-frequency vibrational modes $6,8,13,15$. We follow the excited-state intramolecular charge donor-acceptor dynamics vibrationally. Previously, we identified principal high-frequency vibrational signatures of the excited electronic states for both the charge donor and acceptor in the $\mathrm{N}^{4-}$ transient IR (tIR), or 1D EV spectra (see Supplementary Note 2, Supplementary Fig. 4, and Supplementary Table 1$)^{34}$. The symmetric stretching carboxylate vibration $\left(\omega_{3}=1328 \mathrm{~cm}^{-1}\right)$ in the electronic excited state, referred to as $v_{\mathrm{COO}}$, is a spectrally isolated reporter of the charge-accepting dcbpy ligand and is the high-frequency mode of interest here.

Using polarization-selective $2 \mathrm{D} \mathrm{EV}$ spectroscopy of $\mathrm{N} 3^{4-}$ at a single $\tau_{2}$ time delay, we discovered that two excited electronic states with ${ }^{1}$ MLCT character $\left({ }^{1} \mathrm{MLCT}_{\mathrm{A}}\right.$ and $\left.{ }^{1} \mathrm{MLCT}_{\mathrm{B}}\right)$ are vibronically coupled to charge donor and acceptor vibrations within the excited triplet manifold, likely facilitating ultrafast photoexcited charge transfer ${ }^{34}$. We now use $3 \mathrm{D} \mathrm{EV}$ spectroscopy to measure the timedependent modulation of the vibronic couplings between $v_{\mathrm{COO}}$ and both MLCT $_{\mathrm{A}}$ and MLCT $_{\mathrm{B}}$ due to low-frequency vibrational modes throughout the initial $1500 \mathrm{fs}$ of the photochemical reaction. Here, we omit the spin label to reflect that $\mathrm{MLCT}_{\mathrm{A}}$ and $\mathrm{MLCT}_{\mathrm{B}}$ are strongly spin-mixed states, which is expected from computational work on similar complexes 8,10 and supported in our data by a consistent $\omega_{1}$ peak maxima of $v_{\mathrm{COO}}$ (Supplementary Note 8 and Supplementary Fig. 13). The ground-state bleach (GSB, positive signal) and excited-state absorption (ESA, negative signal) of the carboxylate region are shown in the $2 \mathrm{D}$ EV spectra (Fig. 2a) with the $\mathrm{MLCT}_{\mathrm{A}}, \mathrm{MLCT}_{\mathrm{B}}$, and $v_{\mathrm{COO}}$ transitions highlighted.

$\tau_{2}$-Dependent amplitude oscillations in 2D EV spectra. Nontrivial 2D EV amplitude modulations are observed over the initial $1500 \mathrm{fs}$ of the $\tau_{2}$ delay. The oscillatory amplitude of the 2D EV peak for $v_{\mathrm{COO}}$ vibronically coupled with $\mathrm{MLCT}_{\mathrm{B}}$ is highlighted in Fig. 2a. To observe the amplitude modulations more clearly, the exponential population kinetics are subtracted to obtain the residual amplitude of the integrated $2 \mathrm{D}$ area $\left(\omega_{1}, \omega_{3}\right)$ for the greatest $\sim 10-15 \%$ signal of the $2 \mathrm{D}$ EV peaks measuring the $v_{\mathrm{COO}}$ vibronic coupling with $\mathrm{MLCT}_{\mathrm{A}}$ and $\mathrm{MLCT}_{\mathrm{B}}$ (gray in Fig. 2b, e, respectively; see Supplementary Fig. 7 and Supplementary Table 2). In accord with previously reported wave packet formation in $\mathrm{N} 3^{12}$, two different $\tau_{2}$ ranges of the residuals are temporally selected (red and blue; Fig. 2b, e), and Fourier transformed to identify the principal low-frequency components modulating the 2D EV signal within those $\tau_{2}$ ranges (Fig. 2b-g; Supplementary Fig. 7). The details of the FT analysis are given in Supplementary Note 3. Careful consideration of instrument noise is required, as the oscillations are weak compared with the population decay (Supplementary Fig. 5). Hence, we only consider the $\omega_{2}$ features most prominently above experimental noise (i.e., $v_{\mathrm{Ru}-\mathrm{N}}$ and $v_{\mathrm{Ru} \text {-bpy }}$ ) in Fig. $2 \mathrm{c}, \mathrm{d}, \mathrm{f}, \mathrm{g}$. The noise floor is set by performing the identical FT analysis on the 2D EV spectral region, where the signal level is $\leq 5 \%$ of the maximum 2D EV signal (Supplementary Note 3 and Supplementary Fig. 6). We exclude features at $\omega_{2} \geq 790 \mathrm{~cm}^{-1}$ given our $833 \mathrm{~cm}^{-1}$ Nyquist sampling limit and the features approaching $0 \mathrm{~cm}^{-1}$ (DC limit) due to imperfect population kinetics subtraction. During early relaxation times $\left(0<\tau_{2}<600 \mathrm{fs}\right.$, Fig. $2 \mathrm{c}$, f), a peak at $\omega_{2}=$ $340 \mathrm{~cm}^{-1}$ is measured only for the $v_{\mathrm{COO}}$ vibration of $\mathrm{MLCT}_{\mathrm{B}}$ character. The electronically-localized nature of this excited vibrational wave packet reported here reveals a stricter electronic characterization of this previously reported wave packet ${ }^{12}$. At later relaxation times $\left(400<\tau_{2}<1500 \mathrm{fs}\right)$, a low-frequency vibrational mode at $\omega_{2} \cong 742 \mathrm{~cm}^{-1}$ is clearly coupled to the $v_{\mathrm{COO}}$ vibration of both $\mathrm{MLCT}_{\mathrm{A}}$ and $\mathrm{MLCT}_{\mathrm{B}}$ character. These spectra show a markedly different vibrational and electronic character of the measured coherences between the early and later relaxation periods.

Previous calculations ${ }^{34}$ of the lowest energy triplet state vibrational spectrum of $\mathrm{N}^{4-}$ (Supplementary Note 9 and Supplementary Fig. 14) and resonance Raman experiments ${ }^{42,43}$ aid us in assigning the $340 \mathrm{~cm}^{-1}$ mode and the $742 \mathrm{~cm}^{-1}$ mode as involving the stretching coordinate between the Ru and the dcbpy nitrogens (respectively called $v_{\mathrm{Ru}-\mathrm{N}}$ and $v_{\mathrm{Ru} \text {-bpy }}$ here), with the $v_{\mathrm{Ru} \text {-bpy }}$ primarily localized to the dcbpy ring. A recent surfacehopping trajectory calculation on $\left[\mathrm{Ru}(\mathrm{bpy})_{3}\right]^{2+}$, a molecule similar to $\mathrm{N}^{8}$, included on-the-fly, spin-orbit coupling and found low-frequency vibrations analogous to $v_{\mathrm{Ru}-\mathrm{N}}$ and $v_{\mathrm{Ru}-b p y}$ promoting ultrafast ISC due to excited-state mixing. To understand the ligand's role in realizing long-lived charge separation, the relevant electronic and vibrational coordinates and their couplings must be mapped out. From Fig. 2, the vibronic eigenstate basis explored here for $\mathrm{N}^{4-}$ is established and consists of: the excited electronic states $\left(\mathrm{MLCT}_{\mathrm{A}} / \mathrm{MLCT}_{\mathrm{B}}\right)$, the chargeaccepting high-frequency vibration $\left(v_{\mathrm{COO}}\right)$, and the lowfrequency vibrations $\left(v_{\mathrm{Ru}-\mathrm{N}}, v_{\mathrm{Ru}-\mathrm{bpy}}\right)$. An effective Hamiltonian for a complicated, multi-coordinate system is distilled down to these measured vibronic eigenstates. We introduce the eigenstates and a density matrix element notation for use in the remaining discussion: $\rho_{a b}\left(\tau_{2}\right)=\left|a\left(\tau_{2}\right)\right\rangle\left\langle b\left(\tau_{2}\right)\right|$ specifies a density matrix element, and $a, b \in\{1-8\}$ are the numbered vibronic eigenstates given in Fig. 3 (e.g., the $v_{\mathrm{Ru}-\mathrm{N}}$ coherence is represented: $\left.|B ; 0,0,0\rangle\langle B ; 1,0,0|=\rho_{35}\right)$.

The early-time excited-state vibrational wave packet. The $3 \mathrm{D}$ EV experiment provides a more complete description of the vibronic states involved in the coherent superposition composing the MLCT $_{\mathrm{B}}$ excited-state vibrational wave packet measured at $\omega_{2}=$ $340 \mathrm{~cm}^{-1}$. The red $\omega_{2}$ spectrum in Fig. $2 \mathrm{f}$ demonstrates that the $2 \mathrm{D}$ EV peak intensity oscillations at early times arise from the $\rho_{35}$ density matrix element. We characterize the time-dependence of the frequencies in the vibrational wave packet by the spectral phase, $\phi\left(\omega_{2}\right)=\tan ^{-1}\left(\frac{\operatorname{Im}\left[\mathrm{FT}\left(S\left(\tau_{2}\right)\right)\right]}{\operatorname{Re}\left[\operatorname{FT}\left(S\left(\tau_{2}\right)\right)\right]}\right)$, where $S\left(\tau_{2}\right)$ is the $2 \mathrm{D} \mathrm{EV}$ residual signal during $0<\tau_{2}<600$ fs for $\mathrm{MLCT}_{\mathrm{B}}$ shown in Fig. 2e (red). The group delay is obtained from the spectral phase, and is defined as $\tau_{d}\left(\omega_{2}\right)=-\mathrm{d} \phi\left(\omega_{2}\right) / \mathrm{d} \omega_{2}$. The spectral phase contains time versus 
a
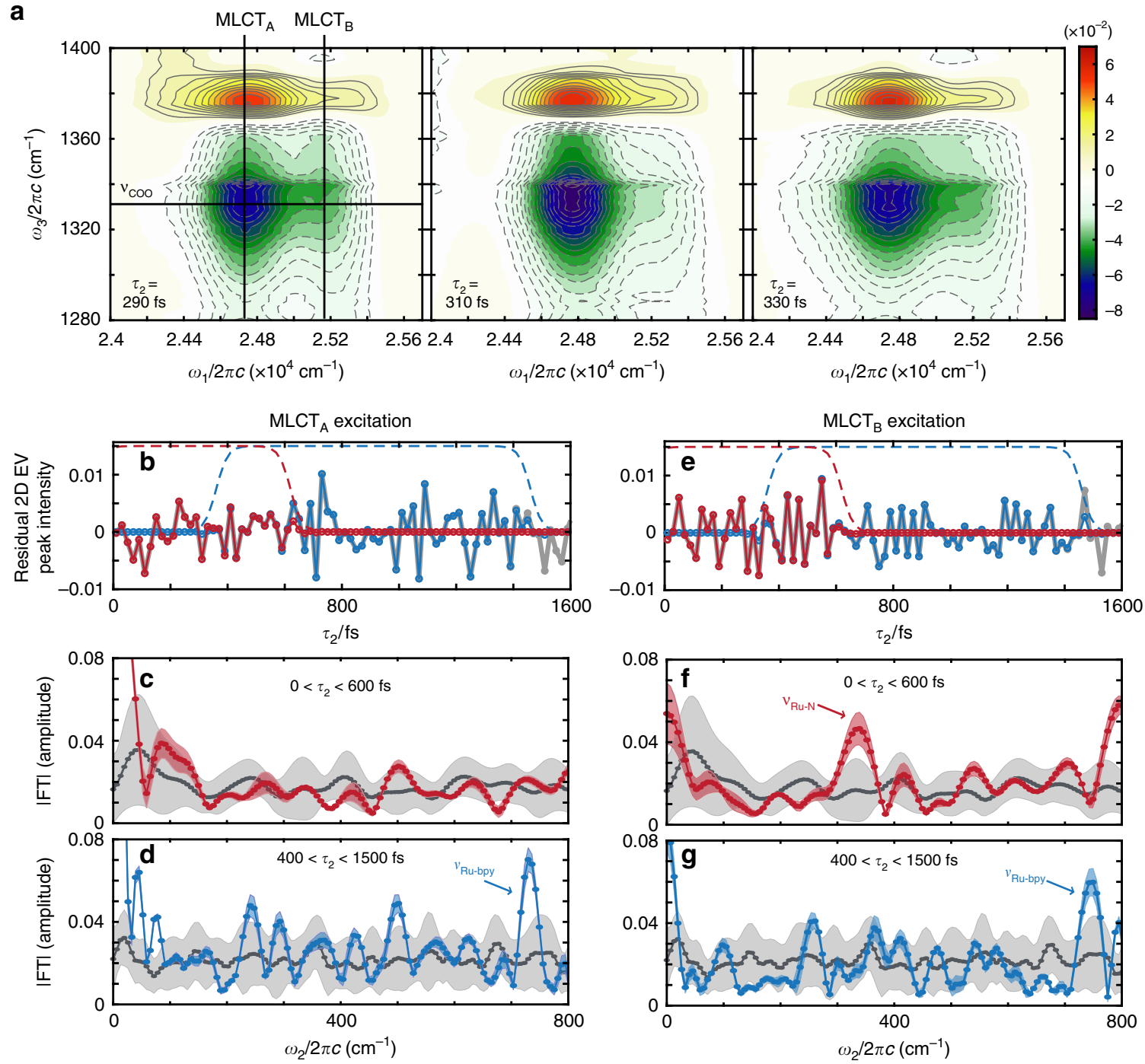

Fig. 2 Low-frequency amplitude modulations in two-dimensional electronic-vibrational (2D EV) spectra. a $\tau_{2}$-dependent 2D EV spectra show oscillatory amplitude; notably, the $\nu_{\mathrm{COO}}\left(\omega_{3}=1328 \mathrm{~cm}^{-1}\right)$ excited-state vibration characterized by $\mathrm{MLCT}_{\mathrm{B}}\left(\omega_{1} \cong 25,200 \mathrm{~cm}^{-1}\right)$ excitation. Contour lines: solid (dashed) $=$ positive (negative) signal, $5 \%$ intervals beginning at $\pm 10 \%$. Residual intensity of the integrated $\left(\omega_{1}, \omega_{3}\right) 2 \mathrm{D}$ peak area for the $\nu_{\mathrm{COO}}$ coupled to the (b) $\mathrm{MLCT}_{\mathrm{A}}\left(\omega_{1} \cong 24,700 \mathrm{~cm}^{-1}\right)$ and $(\mathbf{e}) \mathrm{MLCT}_{\mathrm{B}}$ electronic excited states (gray, circles). Temporal filtering (dashed) isolates early relaxation times ( $0<$ $\tau_{2}<600 \mathrm{fs}$, red) or later relaxation times $\left(400<\tau_{2}<1500 \mathrm{fs}\right.$, blue) to identify transient, low-frequency amplitude modulations in either time range. Fourier Transform (FT) spectra of the temporally filtered $\left[(\mathbf{c})\right.$ early = red; (d) later = blue $2 \mathrm{D}$ residuals of $\mathrm{MLCT}_{\mathrm{A}}$ excitation show principal low-frequency components (i.e., $\nu_{\text {Ru-N }}$ and/or $\nu_{\text {Ru-bpy }}$ ) responsible for the $2 \mathrm{D}$ EV peak amplitude modulation. Corresponding FT spectra for $\mathrm{MLCT}_{\mathrm{B}}$ excitation are shown in (f) and (g). In all FT spectra, the gray spectra are the background (no EV signal); the shaded regions represent \pm 1 standard deviation from the average data (shown by circles). See Supplementary Note 3 for discussion of data processing. The number of $\left(\omega_{1}, \omega_{3}\right)$ points: $M_{L C T}=45 ; M_{A} L_{B}=24 ; B a c k g r o u n d$ $($ signal $\leq 5 \% \max )=3295$.

frequency information of a wave packet; for example, a quadratic variation in $\phi(\omega)$ corresponds to a linear group delay because the frequencies of the wave packet are changing linearly in time (see Supplementary Note 4 and Supplementary Fig. 8a, b). We note that the extracted group delay (Fig. 4, green), resulting from the best fit of the spectral phase, is positive and predominantly quadratic within the $\omega_{2}$ region defined by the spectral FWHM (gray arrows). Interestingly, this form of the group delay describes the wave packet shifting to higher frequencies $\left(300 \mathrm{~cm}^{-1} \rightarrow 370 \mathrm{~cm}^{-1}\right.$ over 140 $\mathrm{fs} \rightarrow 520 \mathrm{fs}$ in $\tau_{\mathrm{d}}$ ) in time. The blue-shift of the vibrational frequencies as a function of $\tau_{2}$ is also revealed through a sliding window short-time Fourier transform (STFT) (Supplementary Fig. 9). We attribute the change in vibrational frequencies to the nonequilibrium relaxation of the excited wave packet with respect to the $v_{\mathrm{Ru}-\mathrm{N}}$ coordinate measured experimentally during $0<\tau_{2}<$ $600 \mathrm{fs}$. This dynamic blue-shift is suggestive of a rapid vibrational relaxation of the highly excited $v_{\mathrm{Ru}-\mathrm{N}}$ coordinate on $\mathrm{MLCT}_{\mathrm{B}}$ down the multidimensional anharmonic potential of the $v_{\mathrm{Ru}-\mathrm{N}}$. Similar observations of blue-shifting of vibrational modes on photoexcited multidimensional surfaces have been made in organic complexes ${ }^{44}$ and in reports of bridging cyanide ligand vibrational relaxation in mixed-valence complexes during photoexcited charge transfer ${ }^{45}$. Consistent with earlier studies ${ }^{12}$, the initial wave packet has largely disappeared by $600 \mathrm{fs}$ as shown by the absence of an $\omega_{2}=340 \mathrm{~cm}^{-1}$ peak in Fig. 2d, g and it reflects the diminishing of the $\rho_{35}$ density matrix element, triggering subsequent electronic delocalization. 

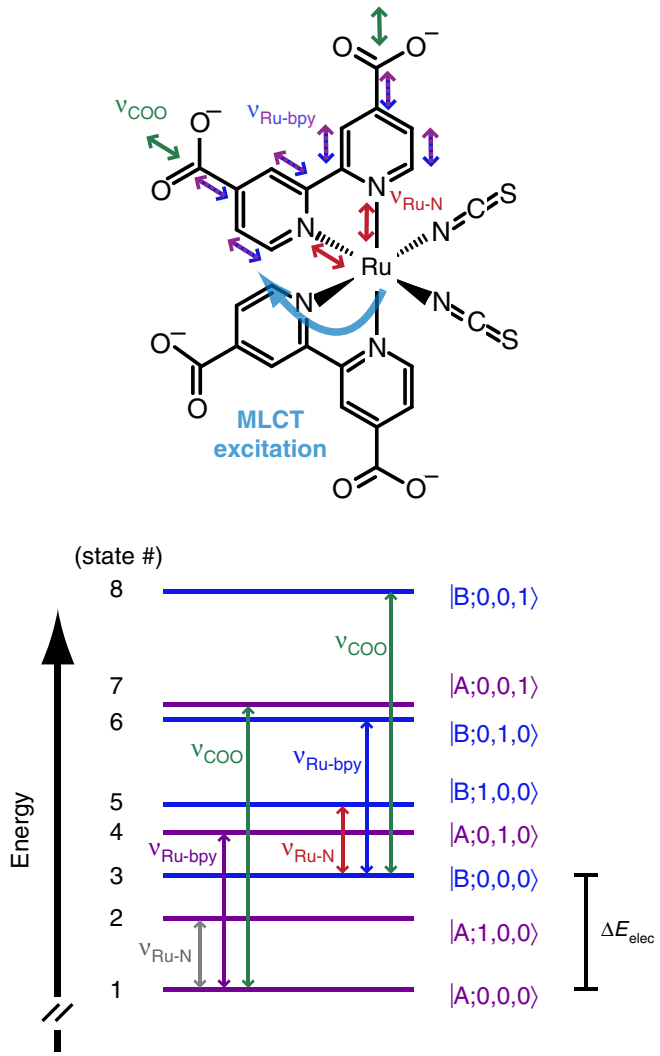

Fig. 3 Vibronic eigenstate basis and energy-level diagram. The measured eigenstate basis of $\mathrm{N}^{4-}$ is cast in terms of $\mid$ elec; $\left.v_{\mathrm{Ru}-\mathrm{N}}, v_{\mathrm{Ru}-\mathrm{bpy}}, v_{\mathrm{COO}}\right\rangle$, where elec $=A\left(M L C T_{A}\right.$, purple $)$ or $B\left(M L C T_{B}\right.$, blue $)$ and the three following quantum numbers relate the quanta in each of the vibrational modes listed in increasing transition energy. The relative energies are derived from the multidimensional $\mathrm{EV}$ measurements: $\Delta E_{\text {elec }} \cong 500 \mathrm{~cm}^{-1}$ from $\omega_{1} ; \nu_{\mathrm{Ru}-\mathrm{N}}=340 \mathrm{~cm}^{-1}$ and $\nu_{\mathrm{Ru}-\mathrm{bpy}}=742 \mathrm{~cm}^{-1}$ from $\omega_{2} ;$ and $\nu_{\mathrm{COO}}=$ $1328 \mathrm{~cm}^{-1}$ from $\omega_{3}$. The eigenstates are numbered in increasing energy for reference throughout the discussion. The $\mathrm{N}^{4-}$ structure (top) schematically depicts the electronic excitation (light blue) and the vibrational motions involved with photoexcited charge transfer. The vibrational mode arrow colors correspond to the respective transitions given in the energy-level diagram below.

Vibronic coherence aids nonadiabatic internal conversion. The later time $\left(400<\tau_{2}<1500 \mathrm{fs}\right)$ dynamics utilize different lowfrequency vibrational mode couplings with the vibronic states. The $\omega_{2} \cong 742 \mathrm{~cm}^{-1}$ features in Fig. $2 \mathrm{~d}$, g demonstrate that $v_{\mathrm{Ru} \text {-bpy }}$ has a time-dependent coupling with $v_{\mathrm{COO}}$ and participates in the $\rho_{14}$ and $\rho_{36}$ coherences. The $v_{\text {Ru-bpy }}$ mode also changes the Ru-N (of dcbpy) distance, emphasizing the importance of this coordinate for ultrafast relaxation in $\mathrm{N}^{4-}{ }^{4}$. Since the $\omega_{2}$ spectra (Fig. 2) lack dynamical information occurring on shorter timescales within the $400<\tau_{2}<1500 \mathrm{fs}$ period, a careful time-frequency analysis $^{25}$ (Fig. 5a,b) is used to resolve oscillatory electronic character of the coherences involving $v_{\mathrm{Ru} \text {-bpy. }}$. Proper selection of the temporal filter used in our analysis is required to extract the transient $v_{\mathrm{Ru} \text {-bpy }}$ dynamics and reasonably interpret the result (Supplementary Note 6). Importantly, the $\omega_{2}$ spectra during the later time period $\left(400<\tau_{2}<1500 \mathrm{fs}\right.$, Fig. $2 \mathrm{~d}$, g) show that the data sufficiently resolve only one low-frequency mode coupling: the $v_{\text {Ru-bpy }}$ mode at $742 \mathrm{~cm}^{-1}$ which has a vibrational period of $45 \mathrm{fs}$. This is a case where a reliable time-frequency analysis is obtainable with a sliding window STFT method ${ }^{46}$. We use a temporal window that targets the presence of $45 \mathrm{fs}$ oscillations in the $\tau_{2}$-dependent data to understand the dynamical behavior of

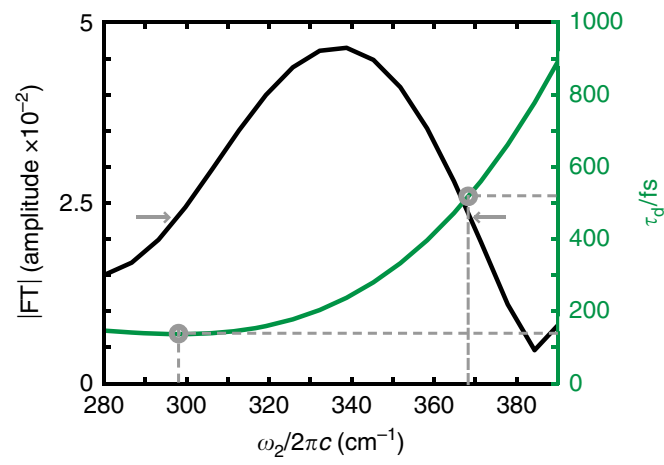

Fig. 4 Early MLCT relaxation dynamics: electronically localized excitedstate vibrational wave packet. The $\omega_{2}$ spectral amplitude (black; FWHM= gray arrows) of the $\mathrm{MLCT}_{\mathrm{B}}$ excited-state vibrational wave packet arises due to coherence between the $\nu_{\mathrm{COO}}$ and one quantum of a Ru-N stretching mode $\left(\nu_{\mathrm{Ru}-\mathrm{N}}=340 \mathrm{~cm}^{-1}\right)$ within the excited-state manifold. The $\tau_{\mathrm{d}^{-}}$ dependence of the $\omega_{2}$ frequencies composing the wave packet (green) indicate blue-shifting as the wave packet propagates. Although the analytical form of $\tau_{d}\left(\omega_{2}\right)$ depends heavily on the polynomial function used to fit $\phi\left(\omega_{2}\right)$, the blue-shifting behavior of the wave packet is consistent across many functions. See Supplementary Note 4, Supplementary Fig. 8, and Supplementary Table 3 for spectral phase fits.

the $v_{\mathrm{Ru} \text {-bpy }}$ mode (see Supplementary Note 6 and Supplementary Figs. 11, 12 for extensive discussion of temporal windowing). We emphasize that our STFT analysis provides a stand-alone interpretation of the data, because only one low-frequency mode is relevant in our later time data as identified in Fig. $2 \mathrm{~d}$, g, which is independent of the time-frequency analysis used in Fig. 5. It is worthwhile to note here that the STFT method becomes less suitable as multiple frequency components contribute to a timedependent signal of interest. This is because no single temporal window can be optimal for resolving many different frequency components in a time-frequency analysis, especially if they vary significantly in frequency and transient behavior. For those complicated cases, more advanced time-frequency transforms are required for reliable interpretation ${ }^{46}$. From our STFT analysis, a vibronic coherence is uncovered persisting throughout this later $\sim 1$ ps of relaxation, which facilitates nonadiabatic IC.

The $\rho_{36}$ coherence appears (Fig. 5b, blue) as the initially excited wave packet diminishes. Thereafter, the $\rho_{36}$ coherence transfers to $\rho_{14}$-changing in electronic character from $\mathrm{MLCT}_{\mathrm{B}}$ to $\mathrm{MLCT}_{\mathrm{A}}$ by $\tau_{2} \cong 650-700 \mathrm{fs}$. This oscillatory dynamic continues for $\sim 1 \mathrm{ps}$, as the amplitude of the $\rho_{36}$ and $\rho_{14}$ coherences oscillate out-of-phase with periodicity of $340 \pm 40 \mathrm{fs}$. We simulate the time-dependent behavior of $\rho_{36}$ and $\rho_{14}$ as an isolated two-level system, where a $50 \mathrm{~cm}^{-1}$ nonadiabatic coupling reproduces the $340 \pm 40 \mathrm{fs}$ Rabilike oscillation seen in the experimental data (Fig. 5c; Supplementary Note 7). Here, the observed Rabi-like oscillations result from the vibronic coherence transfer between $\rho_{36}$ and $\rho_{14}{ }^{47}$.

\section{Discussion}

There is a great need for experimental methods that provide unambiguous measurement of vibronic coherences and couplings in order to uncover their role in ultrafast charge and energy transfer ${ }^{5,24,33}$. The results presented above clearly demonstrate the capability of multidimensional EV spectroscopy to directly monitor the temporal evolution of vibronic coherences and couplings during multi-coordinate photochemical processes. The progression of the photoexcited charge transfer process in $\mathrm{N}^{4-}$ measured in this study is summarized in Fig. 6.

The early-time $\rho_{35}$ coherence reveals an impressive degree of intramolecular couplings that propagate for the initial $\sim 600 \mathrm{fs}$. 

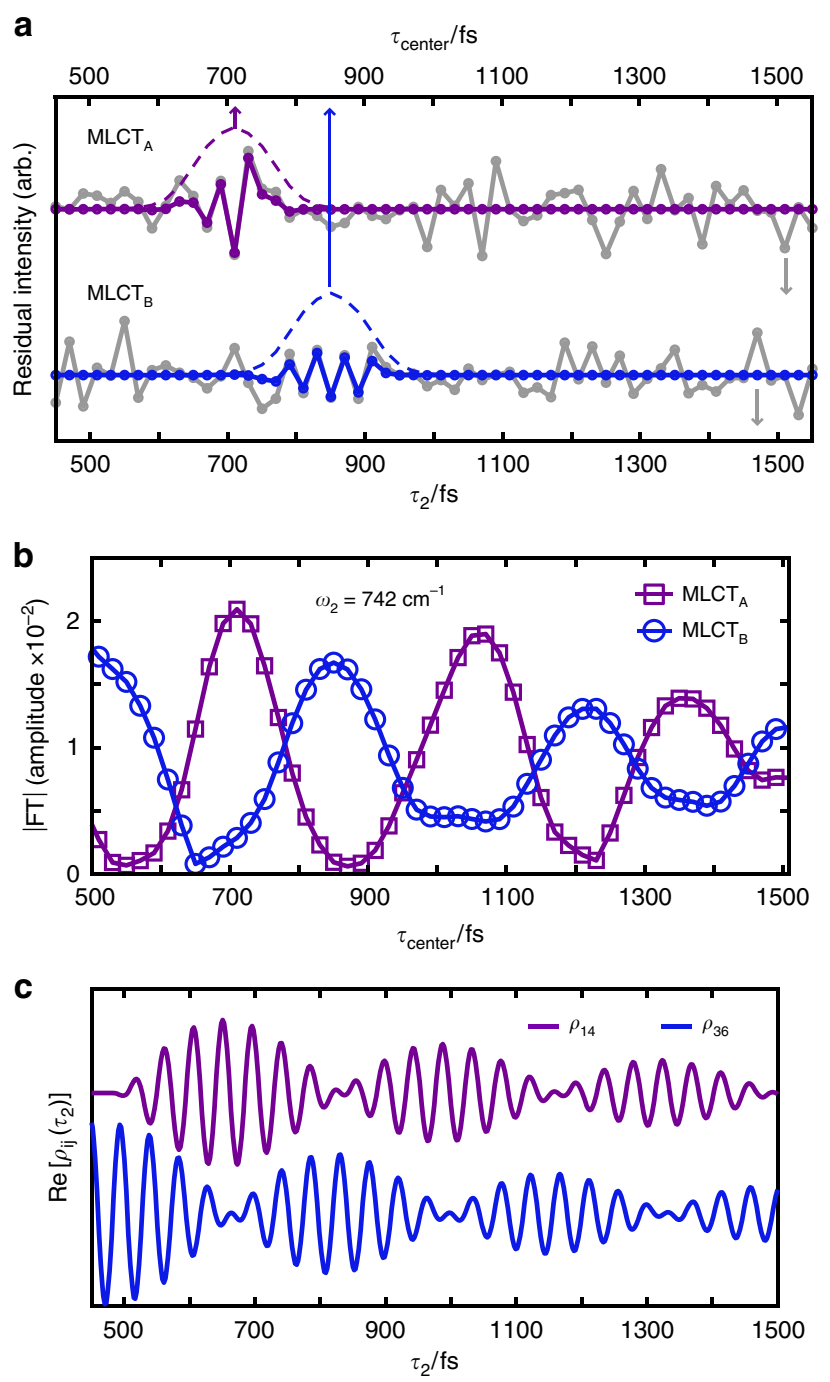

Fig. 5 Later MLCT relaxation dynamics: nonadiabatic internal conversion through vibronic coherence. a Residual intensity (gray) of the integrated ( $\omega_{1}$, $\left.\omega_{3}\right) 2 \mathrm{D}$ peak areas for the $v_{\mathrm{CoO}}$ vibration with $\mathrm{MLCT}_{\mathrm{A}}$ (top) and $\mathrm{MLCT}_{\mathrm{B}}$ (bottom) character; data are offset for plotting. Examples of the temporal filters (purple/blue, dashed) used in short-time FT analysis (FWHM $=120 \mathrm{fs,}$ centered at $\tau_{\text {center }}$ (top axis)) of the $\tau_{2}$-dependent residual intensity. The purple window $\left(\tau_{\text {center }}=710 \mathrm{fs}\right)$ highlights the $\nu_{\text {Ru-bpy }}\left(742 \mathrm{~cm}^{-1}\right)$ coherent beating with $v_{\mathrm{COO}}$ having exclusively $\mathrm{MLCT}_{\mathrm{A}}$ character while at a later delay $\left(\tau_{\text {center }}=850 \mathrm{fs}\right)$ the blue window shows the $\nu_{\text {Ru-bpy }}$ coherence having exclusively $\mathrm{MLCT}_{\mathrm{B}}$ character. b Short-time $\mathrm{FT}$ results reveal the oscillatory electronic character of the $\nu_{\mathrm{Ru} \text {-bpy }}$ coherence signature between $M L C T_{A}$ and $\mathrm{MLCT}_{\mathrm{B}}$ with $340 \pm 40 \mathrm{fs}$ periodicity. c Simulation of the coherence block in the Redfield relaxation tensor for $\rho_{14}$ (purple) and $\rho_{36}$ (blue) during $\tau_{2}$ with an $800 \mathrm{fs}$ coherence decay time where $\omega_{14}=\omega_{36}=742 \mathrm{~cm}^{-1}$ and a $50 \mathrm{~cm}^{-1}$ nonadiabatic coupling reproduces electronic oscillatory behavior for the vibronic coherence involving $\nu_{\text {Ru-bpy }}$ with $M_{L C T_{A}}$ and $M_{\text {LCT }}$ (data offset). See Supplementary Note 5 for discussion of Redfield relaxation dynamics and Supplementary Note 7 for simulation details.

This wave packet is confined to $\mathrm{MLCT}_{\mathrm{B}}$ electronic character, indicating that the Ru-N distance of the $v_{\mathrm{Ru}-\mathrm{N}}$ stretching coordinate specifically modulates the vibronic coupling between $v_{\mathrm{COO}}$ and the $\mathrm{MLCT}_{\mathrm{B}}$ during the initial relaxation of photoexcited $\mathrm{N}^{4-}{ }^{-}$(Fig. 6b, red well). The early-time vibrational wave packet measurement clearly differentiates the nature of the vibronic coupling between either $\mathrm{MLCT}_{\mathrm{A}}$ or $\mathrm{MLCT}_{\mathrm{B}}$ and the $v_{\mathrm{COO}}$, which is a distinction that could not be made from a single $2 \mathrm{D} \mathrm{EV}$, tIR, or transient absorption (TA) experiment. The nature of the initial excited-state wave packet, first reported by Kallioinen ${ }^{12}$ using TA, can now be confidently assigned as an excited-state vibrational coherence between $v_{\mathrm{COO}}$ and $v_{\mathrm{Ru}-\mathrm{N}}$ due to the electronically localized 3D EV signal. Confirmation of the triplet character of this excited-state vibrational wave packet is clear because the signal is detected through the coherence with the triplet state $v_{\mathrm{COO}}$, which answers this outstanding question in the field of N3 photophysics. We note that the known ISC time ( $\leq 100 \mathrm{fs}$ ) leaves the existence of the singlet vibrational wave packet ambiguous because the $v_{\mathrm{Ru}-\mathrm{N}}$ vibrational period is on the same order as ISC, if not longer.

A transition occurs between the early and later relaxation periods as the wave packet arising from the $\rho_{35}$ coherence diminishes and the later time wave packet formed by the $\rho_{36}$ coherence emerges (Fig. $6 \mathrm{~b}$, red to blue well). The fact that this transfer occurs between states of the same electronic character suggests that excited-state vibrational coherence transfer bridges the early-time and later time dynamics; however, many other coordinates could also affect the $\rho_{35}$ coherence. Indeed, we excite only a small window of the ${ }^{1}$ MLCT absorption; the observed dynamics will depend on the electronic structure of the initially excited states and the solvation environment ${ }^{48,49}$. Since our pump center frequency and bandwidth cannot directly excite $\rho_{36}$, this coherence must be accessed through intramolecular energy transfer. Conversely, the $\rho_{14}$ is directly excitable, yet its signature is negligible until after the $\rho_{36}$ coherence has formed (Fig. 5b). Importantly, our probe center frequency and bandwidth is insufficient to collapse the coherences observed during $\tau_{2}$ and excite the $\mathrm{v}=0 \rightarrow 1 \quad v_{\mathrm{COO}}$ vibrational transition required for detecting the ESA at $\omega_{3}=1328 \mathrm{~cm}^{-1}$ (Supplementary Note 5 and Supplementary Table 4). Accounting for the quantum pathways capable of producing the measured oscillatory residuals implies that coherence-to-population transfer ${ }^{50}$ plays an important role in the ultrafast photophysics of $\mathrm{N} 3^{4-}$ (see Supplementary Fig. 10). Together, these dynamics suggest that the vibronic couplings of $\mathrm{MLCT}_{\mathrm{B}}$ function as a gateway for the initially excited vibrational coherence to transition into nonadiabatic IC and continue equilibrating within the excited triplet manifold.

Discovering the out-of-phase oscillations between the $\rho_{36}$ and $\rho_{14}$ coherences is key to characterizing the wave packet evolving during the later relaxation period as an excited-state vibronic coherence (Fig. 6c). By comparison, a purely vibrational coherence, such as $\rho_{35}$, would be confined within a single electronic state. A purely electronic coherence would be expected to appear at the earliest delay times, to dephase within 10s-100s of fs, and to yield $\omega_{2}$ features at the difference frequency between the two electronic states independently of any vibrational coordinate. As we report here for $400<\tau_{2}<1500 \mathrm{fs}$, the amplitude of the vibrational coherence oscillates with the electronic character, it appears after $600 \mathrm{fs}$ of relaxation, and it proceeds for $\sim 1 \mathrm{ps}$. This prolonged coherence is characteristically vibronic, as the expected decoherence time slows when a more complete specification of the system eigenstates occurs; ${ }^{29}$ we directly observe this quantum coherence with multidimensional EV spectroscopy. The $50 \mathrm{~cm}^{-1}$ nonadiabatic coupling extracted from our simulations is consistent with the $340 \pm 40 \mathrm{fs}$ vibronic coherence transfer rate between $\rho_{36}$ and $\rho_{14}$, and the near-complete transfer observed in Fig. $5 \mathrm{~b}$ suggests the system is within the strong coupling regime. The combination of the $2 \mathrm{D} \mathrm{EV}$ experiment to map out vibronic couplings between $v_{\mathrm{COO}}$ and either MLCT $\mathrm{A}$ or $\mathrm{MLCT}_{\mathrm{B}}{ }^{34}$, and the $3 \mathrm{D}$ EV experiment to resolve additional excited-state coherences involving $v_{\mathrm{Ru}-\mathrm{N}}$ and $v_{\mathrm{Ru} \text {-bpy }}$ allow the evolution of photoexcited vibronic coherence to be unambiguously measured. 
a

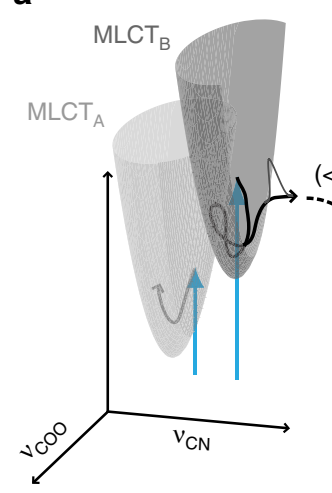

b

Excited state vibrational coherence $\left(100<\tau_{2}<600 \mathrm{fs}\right)$
C

Excited state vibronic coherence $\left(400<\tau_{2}<1500 \mathrm{fs}\right)$

$$
\left(400<\tau_{2}<1500 \mathrm{fs}\right)
$$

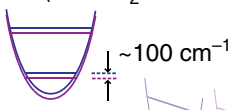
$(<100 \mathrm{fs})$
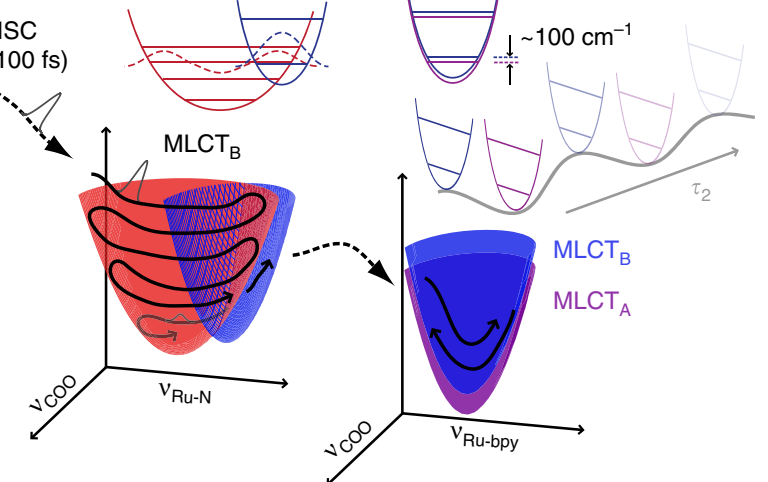

Fig. 6 Coherence evolution during the relaxation of photoexcited N34-- A schematic representation of the first two picoseconds of photoexcited relaxation

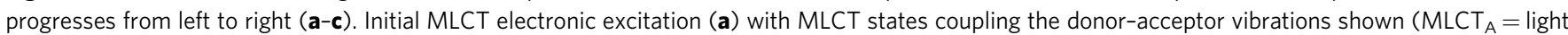
gray well; $\mathrm{MLCT}_{\mathrm{B}}=$ dark gray well) and the initial wave packet passing through ultrafast ISC. The $\sim 600$ fs propagation of the initial excited-state vibrational coherence $\rho_{35}$ (b) involving $\nu_{\mathrm{Ru}-\mathrm{N}}$ of $\mathrm{MLCT}_{\mathrm{B}}$ (red wells); a coherence transfer to $\nu_{\mathrm{Ru} \text {-bpy }}$ of $\mathrm{MLCT}_{\mathrm{B}}$ (blue wells) is merely suggested with sufficient wavefunction overlap involving the $\nu_{\text {Ru-bpy }}$ lowest energy vibration. The $\sim 1$ ps propagation of the excited-state vibronic coherences $\rho_{36}$ and $\rho_{14}$ involving $\nu_{\text {Ru- }}$ bpy (c) depicts the nonadiabatic internal conversion between $\mathrm{MLCT}_{\mathrm{A}}$ (purple) and $\mathrm{MLCT}_{\mathrm{B}}$ (blue) through the $50 \mathrm{~cm}^{-1}$ nonadiabatic coupling strength.

Progress toward tackling the long-standing challenge of characterizing nonequilibrated charge transfer mechanisms in photoexcited donor-acceptor complexes is achieved in this study. Our 3D EV measurements provide an account of how intramolecular charge transfer occurs in an archetypal photosensitizer, detailing the complex interplay between valence electron density and molecular structural dynamics on the fs timescale. We have shown that the cascading relaxation through the nonequilibrated MLCT manifold of $\mathrm{N}^{4-}$ is heavily influenced by $\mathrm{Ru}-\mathrm{N}$ (of dcbpy) vibrations, which effectively bridge the charge-donating $\mathrm{Ru}-(\mathrm{NCS})_{2}$ and charge-accepting dcbpy moieties through excitedstate vibronic couplings. We conclude that at least one excitedstate trajectory in aqueous $\mathrm{N}^{4-}$ that facilitates intramolecular charge transfer is initiated through an excited-state vibrational coherence lasting for $\sim 600 \mathrm{fs}$ before transferring to a secondary coherence that is vibronic in nature and promotes nonadiabatic IC proceeding for another $\sim 1$ ps of relaxation. These results illuminate a mechanism for how solvated $\mathrm{N} 3^{4-}$ utilizes excited state, time-dependent vibronic couplings to achieve long-lived charge separation. In addition to a high density of states and strong spin-orbit coupling, we contribute evidence that structural relaxation involving metal-ligand bonding nitrogen atoms strongly influences excited-state mixing and efficient formation of long-lived ${ }^{3}$ MLCT states 6,8 . This mechanistic insight may be particularly useful for designing more efficient and earthabundant light-harvesting complexes ${ }^{51}$, and for harnessing the typically untapped potential of unthermalized photoexcited states to control photochemical efficacy ${ }^{52}$. Following photochemical reactivity from the perspective of specific vibrational coordinates is a unique advantage of multidimensional EV spectroscopy, which can be exploited to identify critical intramolecular coordinates governing nonequilibrium photoexcited processes. Multidimensional EV spectroscopy will be an incisive tool for understanding photoexcited energy and charge transfer mechanisms during complex, multi-coordinate photochemical processes in molecular and material systems.

\section{Methods}

Experimental description. The 2D EV experiment is performed in a similar way to a tIR experiment with a pair of electronic pump pulses responsible for creating an excited state (i.e., the "bra" and "ket" interactions) followed by an IR probe pulse; see Supplementary Note 1 and Supplementary Figs. 1-3 for complete experimental details. The $\tau_{2}$ delay time (the typical pump-probe relaxation time) is fixed, and the delay time $\left(\tau_{1}\right)$ between a pump pulse pair generated in an interferometer is scanned. A FT over $\tau_{1}$ yields the electronic excitation frequency dimension, $\omega_{1}$, while the high-frequency vibrational detection dimension, $\omega_{3}$, is measured using a spectrometer. A single $2 \mathrm{D} \mathrm{EV} \mathrm{spectrum} \mathrm{is} \mathrm{collected} \mathrm{at} \mathrm{a} \mathrm{given} \tau_{2}$ delay time; it describes the electronic-vibrational frequency correlations present between the electronic states excited in $\tau_{1}$ and the high-frequency vibrations probed in $\tau_{3}$. In this way, $2 \mathrm{D} \mathrm{EV}$ directly measures vibronically coupled degrees of freedom. A series of $2 \mathrm{D}$ EV spectra collected over a range of $\tau_{2}$ delays maps the time-dependence of the vibronic couplings resolved in the 2D EV spectrum. As is common to all $2 \mathrm{D}$ spectroscopies, $\tau_{2}$-dependent signal amplitudes measure population dynamics and coherent superpositions that are accessible within the excitation spectral bandwidth of the pump pulses. The UV pump center frequency $\left(\omega_{0}\right)$ and bandwidth used in these experiments are $\omega_{0}=24,900 \mathrm{~cm}^{-1}$ and fullwidth-at-20\%-max $=1400 \mathrm{~cm}^{-1}$; the mid-IR probe center frequency and bandwidth used here are $\omega_{0}=1375 \mathrm{~cm}^{-1}$, full-width-at- $20 \%$-max $=245 \mathrm{~cm}^{-1}$. Amplitude modulations occurring at the difference frequency between the vibronic states involved in the excited $\tau_{2}$ coherences are measured and can be more directly resolved as residual $2 \mathrm{D}$ peak intensity following subtraction of signals from population dynamics (see Supplementary Note 3 for complete details). Another FT over the residual intensities during $\tau_{2}$ reveals a third frequency dimension, $\omega_{2}$, that identifies the lower frequency vibrations and molecular coherences responsible for the amplitude modulations in the 2D EV spectra. Resolving all three frequency dimensions yields a three-dimensional (3D) EV spectrum, which in this study contains no common frequencies among any of the three spectral dimensions. Importantly, this means that our 3D EV spectrum requires a high degree of different intramolecular couplings to view 3D EV signal. Moreover, low-frequency mode couplings in $\omega_{2}$ may be time-dependent, appearing only at certain times during various photoexcited molecular relaxation pathways. The time-dependence of the measured vibronic couplings can be ambiguously defined, and perhaps irresolvable, without a time-frequency analysis such as the sliding window shorttime FT analysis used in this work (see Supplementary Note 6).

Instrumental details. The fundamental output of a Ti:Sapphire regenerative amplifier (Spectra Physics Spitfire XP Pro; $800 \mathrm{~nm}, 4.0 \mathrm{~W}, \sim 40$ femtoseconds (fs), $1 \mathrm{kHz}$ ) is used for these studies. The broadband UV (BBUV) pump is generated by the second harmonic of a spectrally broadened portion of the $800 \mathrm{~nm}$ fundamental beam using a $100 \mu \mathrm{m}$ BBO (Type I) crystal (Newlight Photonics); the spectral broadening method is detailed in Supplementary Note 1. An acousto-optic pulse shaper (Fastlite Dazzler) generates the collinear pump pulse pair $\left(\mathbf{k}_{1}, \mathbf{k}_{2}\right)$, delivering $240 \mathrm{~nJ} /$ pulse focused to a $220 \mu \mathrm{m} 1 / \mathrm{e}^{2}$ diameter at the sample. The pump is mechanically chopped at $500 \mathrm{~Hz}$ to collect differential absorption spectra. The difference frequency between the signal and idler fields of a home-built optical parametric amplifier generates the mid-IR probe, which provides $480 \mathrm{~nJ} /$ pulse focused to a $195 \mu \mathrm{m} 1 / \mathrm{e}^{2}$ diameter at the sample. The isotropic signal is collected by setting the polarization angle between the pump and probe to the magic angle $\left(54.7^{\circ}\right)$ for data collection. The instrument response has subsided by 
$\tau_{2}=180-200$ fs given by the solvent only tIR signal and the rise time of the nonresonant integrated pump-probe signal in a $250 \mu \mathrm{m}$ Si wafer (Supplementary Fig. 2).

\section{Data availability}

The data supporting this study are available from the corresponding author upon reasonable request.

Received: 15 July 2019; Accepted: 7 November 2019;

Published online: 09 December 2019

\section{References}

1. Mirkovic, T. et al. Light absorption and energy transfer in the antenna complexes of photosynthetic organisms. Chem. Rev. 117, 249-293 (2017)

2. Hagfeldt, A. \& Gratzel, M. Molecular photovoltaics. Acc. Chem. Res. 33, 269-277 (2000).

3. Reece, S. Y. et al. Wireless solar water splitting using silicon-based semiconductors and earth-abundant catalysts. Science 334, 645-648 (2011).

4. O'Regan, B. \& Gratzel, M. A low-cost, high-efficiency solar-cell based on dyesensitized colloidal $\mathrm{TiO}_{2}$ films. Nature 353, 737-740 (1991).

5. McCusker, J. K. Electronic structure in the transition metal block and its implications for light harvesting. Science 363, 484-488 (2019).

6. Chergui, M. Ultrafast photophysics of transition metal complexes. Acc. Chem. Res. 48, 801-808 (2015).

7. McCusker, J. K. Femtosecond absorption spectroscopy of transition metal charge-transfer complexes. Acc. Chem. Res. 36, 876-887 (2003).

8. Atkins, A. J. \& González, L. Trajectory surface-hopping dynamics including intersystem crossing in $\left[\mathrm{Ru}(\mathrm{bpy})_{3}\right]^{2+}$. J. Phys. Chem. Lett. 8, 3840-3845 (2017).

9. Mai, S. et al. Quantitative wave function analysis for excited states of transition metal complexes. Coord. Chem. Rev. 361, 74-97 (2018).

10. Penfold, T. J., Gindensperger, E., Daniel, C. \& Marian, C. M. Spin-vibronic mechanism for intersystem crossing. Chem. Rev. 118, 6975-7025 (2018).

11. Capano, G., Penfold, T. J., Chergui, M. \& Tavernelli, I. Photophysics of a copper phenanthroline elucidated by trajectory and wavepacket-based quantum dynamics: a synergetic approach. Phys. Chem. Chem. Phys. 19, 19590-19600 (2017).

12. Kallioinen, J. et al. Photoinduced ultrafast dynamics of Ru(dcbpy $)_{2}(\mathrm{NCS})_{2}-$ sensitized nanocrystalline $\mathrm{TiO}_{2}$ films: the influence of sample preparation and experimental conditions. J. Phys. Chem. B 108, 6365-6373 (2004).

13. Kallioinen, J., Benko, G., Sundstrom, V., Korppi-Tommola, J. E. I. \& Yartsev, A. P. Electron transfer from the singlet and triplet excited states of $\mathrm{Ru}$ (dcbpy $)_{2}(\mathrm{NCS})_{2}$ into nanocrystalline $\mathrm{TiO}_{2}$ thin films. J. Phys. Chem. B 106, 4396-4404 (2002).

14. Pettersson Rimgard, B. et al. Ultrafast interligand electron transfer in cis-[Ru $\left.\left(4,4^{\prime} \text {-dicarboxylate-2,2'-bipyridine }\right)_{2}(\mathrm{NCS})_{2}\right]^{4-}$ and implications for electron injection limitations in dye sensitized solar cells. Chem. Sci. 9, 7958-7967 (2018).

15. Bram, O., Messina, F., El-Zohry, A. M., Cannizzo, A. \& Chergui, M. Polychromatic femtosecond fluorescence studies of metal-polypyridine complexes in solution. Chem. Phys. 393, 51-57 (2012).

16. Zhang, X. et al. Visualizing interfacial charge transfer in ru-dye-sensitized $\mathrm{TiO}_{2}$ nanoparticles using x-ray transient absorption spectroscopy. J. Phys. Chem. Lett. 2, 628-632 (2011).

17. Van Kuiken, B. E. et al. Probing the electronic structure of a photoexcited solar cell dye with transient X-ray absorption spectroscopy. J. Phys. Chem. Lett. 3, 1695-1700 (2012).

18. Asbury, J. B., Hao, E., Wang, Y. Q., Ghosh, H. N. \& Lian, T. Q. Ultrafast electron transfer dynamics from molecular adsorbates to semiconductor nanocrystalline thin films. J. Phys. Chem. B 105, 4545-4557 (2001).

19. Wernet, P. Chemical interactions and dynamics with femtosecond X-ray spectroscopy and the role of X-ray free-electron lasers. Philos. Trans. R. Soc. A 377, 20170464 (2019).

20. Lemke, H. T. et al. Coherent structural trapping through wave packet dispersion during photoinduced spin state switching. Nat. Commun. 8, 15342 (2017).

21. Auböck, G. \& Chergui, M. Sub-50-fs photoinduced spin crossover in $[\mathrm{Fe}$ (bpy) $\left.{ }_{3}\right]^{2+}$. Nat. Chem. 7, 629 (2015).

22. Kraus, P. M., Zürch, M., Cushing, S. K., Neumark, D. M. \& Leone, S. R. The ultrafast X-ray spectroscopic revolution in chemical dynamics. Nat. Rev. Chem. 2, 82-94 (2018).

23. Chen, L. X. et al. Capturing a photoexcited molecular structure through timedomain X-ray absorption fine structure. Science 292, 262-264 (2001)

24. Scholes, G. D. et al. Using coherence to enhance function in chemical and biophysical systems. Nature 543, 647-656 (2017).
25. Meneghin, E. et al. Coherence in carotenoid-to-chlorophyll energy transfer. Nat. Commun. 9, 3160 (2018)

26. Nee, M. J., Baiz, C. R., Anna, J. M., McCanne, R. \& Kubarych, K. J. Multilevel vibrational coherence transfer and wavepacket dynamics probed with multidimensional IR spectroscopy. J. Chem. Phys. 129, 084503 (2008).

27. Butkus, V., Zigmantas, D., Valkunas, L. \& Abramavicius, D. Vibrational vs. electronic coherences in 2D spectrum of molecular systems. Chem. Phys. Lett. 545, 40-43 (2012)

28. Fuller, F. D. et al. Vibronic coherence in oxygenic photosynthesis. Nat. Chem. 6, 706-711 (2014)

29. Jonas, D. M. Vibrational and nonadiabatic coherence in 2D electronic spectroscopy, the Jahn-Teller effect, and energy transfer. Ann. Rev. Phys. Chem. 69, 327-352 (2018).

30. Butkus, V. et al. Discrimination of diverse coherences allows identification of electronic transitions of a molecular nanoring. J. Phys. Chem. Lett. 8, 2344-2349 (2017)

31. Khalil, M., Demirdoven, N. \& Tokmakoff, A. Vibrational coherence transfer characterized with Fourier-transform 2D IR spectroscopy. J. Chem. Phys. 121, 362-373 (2004).

32. Engel, G. S. et al. Evidence for wavelike energy transfer through quantum coherence in photosynthetic systems. Nature 446, 782-786 (2007).

33. Rafiq, S. \& Scholes, G. D. From fundamental theories to quantum coherences in electron transfer. J. Am. Chem. Soc. 141, 708-722 (2018).

34. Gaynor, J. D., Petrone, A., Li, X. \& Khalil, M. Mapping vibronic couplings in a solar cell dye with polarization-selective two-dimensional electronic-vibrational spectroscopy. J. Phys. Chem. Lett. 9, 6289-6295 (2018).

35. Courtney, T. L., Fox, Z. W., Estergreen, L. \& Khalil, M. Measuring coherently coupled intramolecular vibrational and charge-transfer dynamics with twodimensional vibrational electronic spectroscopy. J. Phys. Chem. Lett. 6 , 1286-1292 (2015)

36. Oliver, T. A. A., Lewis, N. H. C. \& Fleming, G. R. Correlating the motion of electrons and nuclei with two-dimensional electronic-vibrational spectroscopy. Proc. Natl Acad. Sci. USA 111, 16628-16628 (2014).

37. Gaynor, J. D. \& Khalil, M. Signatures of vibronic coupling in two-dimensional electronic-vibrational and vibrational-electronic spectroscopies. J. Chem. Phys. 147, 094202 (2017).

38. Bakulin, A. A. et al. Real-time observation of multiexcitonic states in ultrafast singlet fission using coherent 2D electronic spectroscopy. Nat. Chem. 8, 16-23 (2016).

39. Romero, E. et al. Quantum coherence in photosynthesis for efficient solarenergy conversion. Nat. Phys. 10, 676 (2014).

40. Ruetzel, S. et al. Multidimensional spectroscopy of photoreactivity. Proc. Natl Acad. Sci. USA 111, 4764-4769 (2014).

41. Tavernelli, I., Curchod, B. F. E. \& Rothlisberger, U. Nonadiabatic molecular dynamics with solvent effects: A LR-TDDFT QM/MM study of ruthenium (II) tris (bipyridine) in water. Chem. Phys. 391, 101-109 (2011).

42. Shoute, L. C. T. \& Loppnow, G. R. Excited-state metal-to-ligand charge transfer dynamics of a ruthenium(II) dye in solution and adsorbed on $\mathrm{TiO}_{2}$ nanoparticles from resonance Raman spectroscopy. J. Am. Chem. Soc. 125, 15636-15646 (2003).

43. Greijer, H., Lindgren, J. \& Hagfeldt, A. Resonance Raman scattering of a dyesensitized solar cell: mechanism of thiocyanato ligand exchange. J. Phys. Chem. B 105, 6314-6320 (2001).

44. Ishii, K., Takeuchi, S. \& Tahara, T. A 40-fs time-resolved absorption study on cis-stilbene in solution: observation of wavepacket motion on the reactive excited state. Chem. Phys. Lett. 398, 400-406 (2004).

45. Lynch, M. S., Slenkamp, K. M. \& Khalil, M. Communication: probing nonequilibrium vibrational relaxation pathways of highly excited $\mathrm{CN}$ stretching modes following ultrafast back-electron transfer. J. Chem. Phys. 136, 241101 (2012).

46. Volpato, A. \& Collini, E. Time-frequency methods for coherent spectroscopy. Opt. Express 23, 20040-20050 (2015).

47. Rury, A. S. \& Dawlaty, J. M. Rabi-like vibrational coherence transfer in a hydrogen-bonded charge transfer material. Phys. Rev. B 95, 214309 (2017)

48. Durrant, J. R. et al. The excitation wavelength and solvent dependance of the kinetics of electron injection in $\mathrm{Ru}(\mathrm{dcbpy})_{2}(\mathrm{NCS})_{2}$ sensitized nanocrystalline $\mathrm{TiO}_{2}$ films. Z. Phys. Chem. 212, 93-98 (1999).

49. Benko, G., Kallioinen, J., Korppi-Tommola, J. E. I., Yartsev, A. P. \& Sundstrom, V. Photoinduced ultrafast dye-to-semiconductor electron injection from nonthermalized and thermalized donor states. J. Am. Chem. Soc. 124, 489-493 (2002)

50. May, V. \& Kuhn, O. Charge and Energy Transfer Dynamics in Molecular Systems, 2nd edn (Wiley, Weinheim, 2004).

51. Chábera, P. et al. A low-spin Fe ${ }^{(\mathrm{III})}$ complex with 100-ps ligand-to-metal charge transfer photoluminescence. Nature 543, 695 (2017).

52. Grumstrup, E. M., Johnson, J. C. \& Damrauer, N. H. Enhanced triplet formation in polycrystalline tetracene films by femtosecond optical-pulse shaping. Phys. Rev. Lett. 105, 257403 (2010). 


\section{Acknowledgements}

The development of 2D EV spectroscopy is supported by the National Science Foundation (NSF) (Grant no. CHE 1565759). J.D.G. is supported by the NSF GRFP, Division of Graduate Education (No. DGE-1256082).

\section{Author contributions}

J.D.G., J.S., and M.K. designed the experiments. J.D.G. and J.S. performed the experiments. J.D.G. analyzed the data. J.D.G., J.S., and M.K. wrote the paper.

\section{Competing interests}

The generation of the near UV pump pulse used in the experiment is the subject of a US provisional patent application. The relevant information is given below: Provisional Patent Application 62/897,032 filed 9/6/2019 Entitled: "Efficient Generation of Stable sub-20 fs $400 \mathrm{~nm}$ Pulses for High Order Nonlinear Spectroscopy" Inventors: Munira Khalil, James Gaynor, Joel Leger, Jason Sandwisch.

\section{Additional information}

Supplementary information is available for this paper at https://doi.org/10.1038/s41467019-13503-9.

Correspondence and requests for materials should be addressed to M.K.
Peer review information Nature Communications thanks Tobias Brixner, Graham Fleming, and the other, anonymous, reviewer for their contribution to the peer review of this work. Peer reviewer reports are available.

Reprints and permission information is available at http://www.nature.com/reprints

Publisher's note Springer Nature remains neutral with regard to jurisdictional claims in published maps and institutional affiliations.

(c) (i) Open Access This article is licensed under a Creative Commons Attribution 4.0 International License, which permits use, sharing, adaptation, distribution and reproduction in any medium or format, as long as you give appropriate credit to the original author(s) and the source, provide a link to the Creative Commons license, and indicate if changes were made. The images or other third party material in this article are included in the article's Creative Commons license, unless indicated otherwise in a credit line to the material. If material is not included in the article's Creative Commons license and your intended use is not permitted by statutory regulation or exceeds the permitted use, you will need to obtain permission directly from the copyright holder. To view a copy of this license, visit http://creativecommons.org/ licenses/by/4.0/.

(C) The Author(s) 2019 